\title{
Advances in Soft X-ray Tomography
}

Venera Weinhardt $^{1}$, Jian-Hua Chen ${ }^{2}$, Axel Ekman ${ }^{2}$, Mark A. Le Gros ${ }^{1}$, Gerry Mc Dermott ${ }^{2}$ and Carolyn Larabell $^{1}$

${ }^{1}$ Molecular Biophysics and Integrated Bioimaging Division, Lawrence Berkeley National Laboratory, Berkeley, California, United States, ${ }^{2}$ Lawrence Berkeley National Laboratory and UCSF, Berkeley, California, United States

Soft x-ray tomography (SXT) has emerged as a unique method for three-dimensional (3D) imaging of single cells [1,2]. Soft x-rays from 284 to $543 \mathrm{eV}(2.34$ to $4.4 \mathrm{~nm})$ generate contrast for cells in their native, wet state without the use of chemical fixation or staining. In this so-called "water window" energy range, absorption of photons by molecular building blocks, in particular carbon and nitrogen, prevails while water is nearly transparent. Over the last decade, several groups around the world including ours designed, built and applied x-ray microscopes for 3D imaging of bacteria, yeast, algae, plankton, protozoa, viruses, and immortalized and primary cells [3,4]. This presentation will focus on describing recent advances in instrumentation, acquisition strategies, and novel application directions of SXT.

Significant advances in instrumentation are necessary to accommodate the remarkable diversity of biological cells, and the imaging capabilities of SXT setups have been recently pushed to high spatial resolutions [5], switchable resolution modes [6], and alternative acquisition schemes, such as a combination of through-focus deconvolution [7,8], tomographic acquisition approaches [9] and local and half-acquisition tomography. With 5 minutes per tomogram at the Advanced Light Source (ALS), Berkeley, USA, SXT has been applied to image 3D phenotypes of yeast with thousands of cells imaged to analyze the nuclear envelope expansion in budding yeast [10].

To couple structural information with function of specific proteins, several groups have started to work on correlative imaging with confocal [11, 12] and super-resolution fluorescence imaging [13]. The example in the Figure below shows how simplified and automatic approaches are used to correlate the protein expression mapped by cryo fluorescence tomography and the 3D structure collected by SXT at the ALS of membrane-less organelles (stress granules) in osteoblast cells. As some of these organelles are suggested to consist of hierarchical subcompartments, we imaged the RNA partitioning in vitro. Beyond high-resolution visualization, soft x-ray tomography provides a quantitative measure of physico-chemical properties, such as the droplet's viscosity, composition and size.

As an important new development, the imaging of single cells and for emerging directions such as protein phase separation in in vitro and in vivo systems, nanodrugs and artificial cells is now becoming accessible for systematic studies by the development of soft $\mathrm{x}$-ray microscopes and sources for laboratories both as self-built $[14,15]$ as well as commercial systems (SiriusXT). 

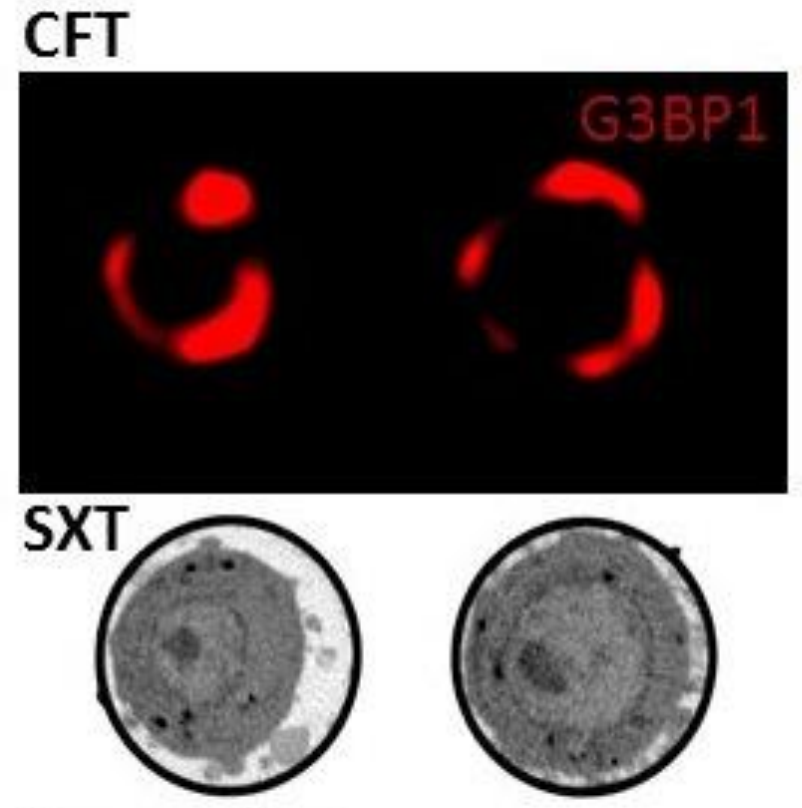

Correlated

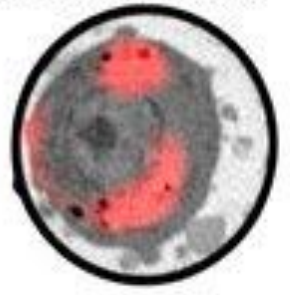

\section{D view}

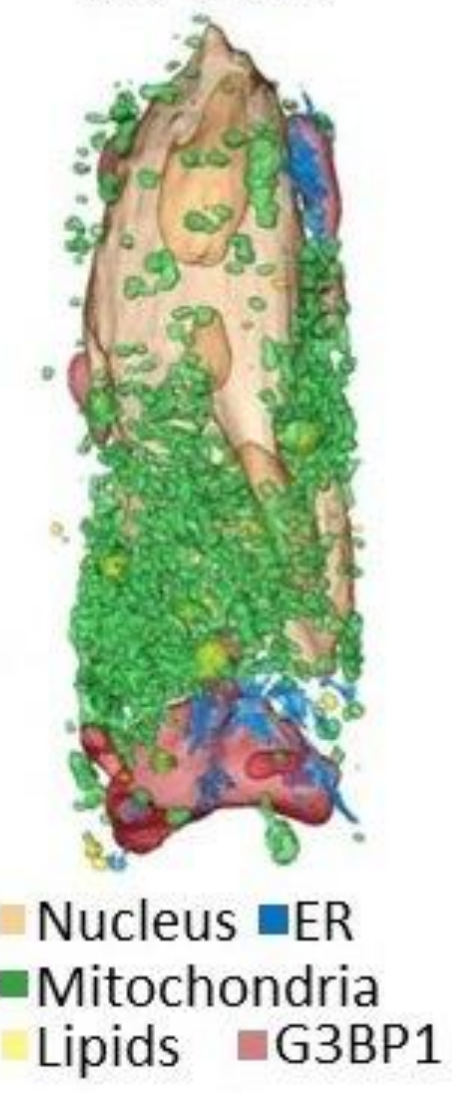

Figure 1. To induce formation of stress granules osteoblast cells (U2OS) were exposed to $0.5 \mu \mathrm{M}$ OsO4. Soft x-ray tomography and cryo fluorescence tomography (CFT) data is automatically correlated to the same orientation and voxel size shown in 3D visualisation of organelles and stress granules.

References

1 Jacobsen, C.,Trends in Cell Biology 9.2 (1999): 44-47.

2 McDermott, G., et al., Bioessays 34.4 (2012): 320-327.

3 Harkiolaki, M., et al., Emerging Topics in Life Sciences 2.1 (2018): 81-92.

4 Weinhardt, V., et al., Biochemical Society Transactions 47.2 (2019): 489-508.

5 Schneider, G., et al., Nature methods 7.12 (2010): 985-987.

6 Weinhardt, V., et al., PLoS One 15.1 (2020): e0227601.

7 Li, F., et al., Optics express 25.7 (2017): 7657-7667.

8 Otón, J., et al., Biomedical optics express 7.12 (2016): 5092-5103.

9 Selin, M., et al., Optics letters 40.10 (2015): 2201-2204.

10 Walters, A. D., et al., Molecular biology of the cell 30.1 (2019): 131-145.

11 Le Gros, M. A., et al., Journal of microscopy 235.1 (2009): 1-8.

12 Hagen, C., et al., Journal of structural biology 177.2 (2012): 193-201.

13 Groen, J., et al., Biophysical reviews (2019): 1-9.

14 Wachulak, P. W., et al., EUV and X-ray Optics: Synergy between Laboratory and Space VI. Vol. 11032. International Society for Optics and Photonics, 2019.

15 Fogelqvist, E., et al., Scientific reports 7.1 (2017): 1-8. 